“ (C) 2016 IEEE. Personal use of this material is permitted. Permission from IEEE must be obtained for all other uses, in any current or future media, including

reprinting/republishing this material for advertising or promotional purposes, creating new collective works, for resale or redistribution to servers or lists, or reuse of any copyrighted component of this work in other works." 


\title{
Selecting Optimal EEG Channels for Mental Taks Classification: An Approach using ICA
}

\author{
Rifai Chai, Member, IEEE, Ganesh R. Naik, Senior Member, IEEE, Tuan N. Nguyen, Senior Member, IEEE \\ Sai Ho Ling, Senior Member, IEEE, and Hung T. Nguyen, Senior Member, IEEE \\ Centre for Health Technologies, Faculty of Engineering and Information Technology \\ University of Technology, Sydney, NSW, Australia \\ E-mail: (Rifai.Chai, Ganesh Naik, TuanNghia.Nguyen, Steve.Ling, Hung.Nguyen)@uts.edu.au
}

\begin{abstract}
This paper presents a systematic method to select optimal electroencephalography (EEG) channels for three mental tasks-based brain-computer interface (BCI) classification. A blind source separation (BSS) based on independent component analysis (ICA) methods with its back-projecting of the scalp map was used for selecting the optimal EEG channels. The three mental tasks included: mental letter composing, mental arithmetic and mental Rubik's cube rolling. Based on a power spectral density (PSD), the features of the two-channel EEG data were extracted, and then were classified by Bayesian neural network. The results of the ICA decomposition with the backprojected scalp map showed that the prominent channels could be selected for dominant features from original six EEG channels (C3, C4, P3, P4, O1, O2) to four dominant channels (P3, O1, C4, O2) with the best two EEG channels selection at O1\&C4. Two channel combinations classification yielded to the best two EEG channels of $01 \& C 4$ with an accuracy of $76.4 \%$, followed by P3\&O2 with an accuracy of $74.5 \%$; $\mathrm{P3} \& \mathrm{C} 4$ with an accuracy of $71.9 \%$ and $01 \& 02$ with an accuracy of $70 \%$.
\end{abstract}

Keywords-electroencephalography (EEG); brain-computer interface (BCI); independent component analysis (ICA), Bayesian neural network; scalp map

\section{INTRODUCTION}

An EEG based-BCI research has been popular in the past two decades with its usable BCI applications such as communication and control, motor substitution, entertainment and gaming, motor rehabilitation and recovery and mental state monitoring [1, 2]. A thought-control powered wheelchair is one of the typical communication and control application. At least three commands are required to control a wheelchair including turning left, turning right and moving forward commands [3].

From the state-of-art research, EEG-based BCI can be divided into selective attention and spontaneous based BCI. For using selective attention BCI, it needs external stimuli which might be cumbersome for the users especially severely disabled individuals. On the other hand the spontaneous based BCI, the users initiate the spontaneous mental signals by themselves. One of the examples of the spontaneous based BCI is the mental motor imagery task which basically relies on the sensorimotor rhythms (SMR) by the motor movement tasks imagination such as imagining hand, feet, tongue and hips movement $[4,5]$. The drawbacks for SMR based BCI, there have been reported that SMR tasks defect in severely disabled individuals [6]. Moreover, individuals who have been paralysed for years may be unable to carry out the SMR task effectively. The other option for spontaneous mental signal is based on non-motor imagery mental task [7, 8]. This paper presents the three non-motor imagery mental-task classification related to the three commonly used wheelchair commands (left, right and forward).

In terms of practical aspects, a BCI system with fewer EEG channels which can be able to provide better performance in term of the portability and convenience is preferable. Independent component analysis (ICA) is a popular blind source separation (BSS) technique which can be used for selecting the optimal EEG channels [9, 10]. ICA has a capability for composing the EEG signals into independent brain activities and with its back projection into original EEG channels of the scalp maps. By further analysing the scalp maps projection, the channels selection can be done. As a result, this paper utilizes the ICA for selecting the optimal EEG channels to classify three mental tasks that maps three corresponding wheelchair control commands. The EEG data were collected from the able bodied and patients with tetraplegia. Two EEG channels classification procedure consisted of two stages. First, a power spectral density (PSD) was used to extract significant EEG features, and Bayesian neural network then is used as a classifier [11].

\section{METHODOLOGY}

\section{A. Brain_Computer Interface Block Diagram}

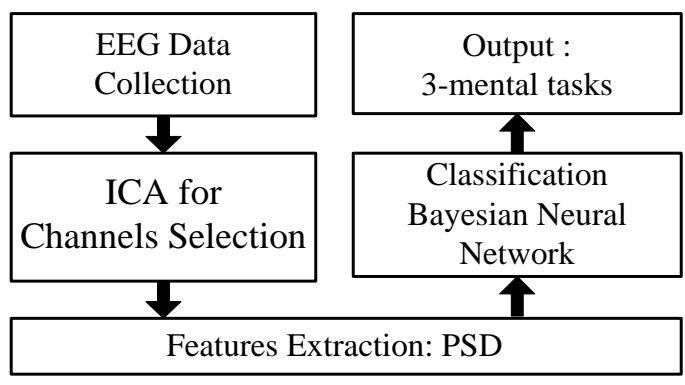

Fig. 1. Block diagram of $\mathrm{BCI}$ in this study 
The proposed block diagram in this study is shown in Fig.1. The process started with the EEG data collection of the mental tasks. The ICA was then utilized for selecting the EEG channels. The software EEGLAB [9] used in the ICA process. After selecting the best EEG channels, features of the EEG data from reduced channels were extracted using PSD and then classified by Bayesian neural network.

\section{B. Data Collection}

The Human Research Ethics Committee of University of Technology, Sydney approved this study of the mental tasks based EEG-BCI experiment with 10 participants, including 5 able-bodied participants and 5 patients with tetraplegia, aged between 25 and 80 years $[8,12]$. With the purpose of the BCI study on severely disabled individuals, the selection of the tetraplegia is in high-level of spinal cord injury (SCI) at $\mathrm{C} 3$, C4, C5 and C6 of the cervical area. The EEG system from Compumedic-Siesta was used with the sampling rate of $256 \mathrm{~Hz}$ and based on the 10-20 electrode system the locations C3, C4, $\mathrm{P} 3, \mathrm{P} 4, \mathrm{O} 1$ and $\mathrm{O} 2$ were selected for EEG channels [13]. As shown in Fig. 2, the left earlobe was used for reference electrode and the right earlobe was used for ground electrode.
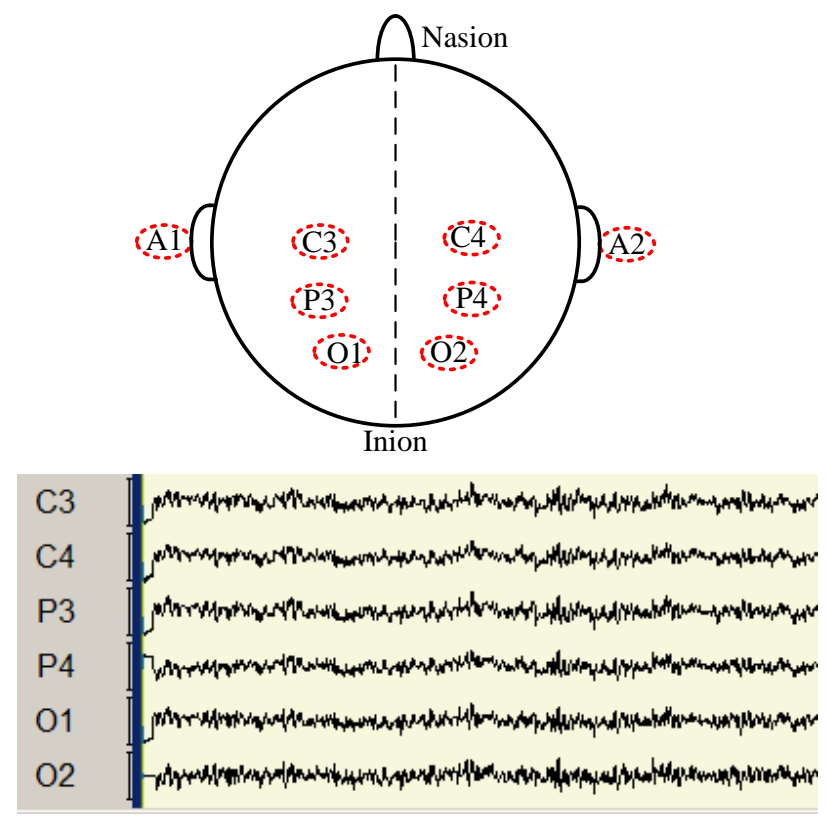

Fig. 2. Six EEG channels used in the experiment

During the EEG experiment, prepping and EEG gels were applied and the electrodes impedances were measured. The impedance was maintained below 5KOhm. Minimum eye blinks and other movement were kept as minimum as possible. Furthermore, data with the strong artifacts' contamination were discarded. Three non-motor imagery mental tasks were used in this experiment including: ( $i$ ) mental letter composing where participants were asked to compose words in their mind; (ii) mental arithmetic where participants were asked to solve multiplication calculation in their mind; (iii) mental Rubik's cube rolling where participants were asked to imagine a figure Rubik's cube rolling forward.
The experiment was repeated for 10 sessions for each mental task with $15 s$ duration. The first $3 s$ of the EEG raw data were discarded as preparation time. As a result, only $12 \mathrm{~s}$ duration of data were used for further signal processing. A $2 s$ moving time window was applied to the raw data with $0.25 \mathrm{~s}$ overlapping segment. Thus, in each mental task, 410 samples of window segments or 4100 samples from 10 participants were collected. The obtained data set was further analysed by ICA method.

\section{Independent Component Analysis}

ICA is one of the popular BSS techniques which finds linear projection of EEG data with the aim of maximizing the mutual independence $[9,14]$. For EEG signal processing, data for the input of the ICA are a matrix of $n$-channels (rows) by $t$ time points (columns) of raw EEG signals. Given linear mixing model $n$-channel scalp EEG signal, $x=\left[x_{1}, x_{2}, \ldots x_{n}\right]$, generated by $m$ independent sources, $s=\left[s_{1}, s_{2}, \ldots s_{\mathrm{n}}\right]$, given in the following equation:

$$
x=A \cdot s
$$

where $A$ denotes the $n \times m$ of the mixing matrix ICA model.

In details, ICA performs a BSS of the data matrix of the EEG signal $(x)$ based only on the criterion of the resulting source time courses which are maximally independent. The recovered source signal $u$ can be found by applying an unmixing matrix $W(m \times n)$ to the EEG data $x$, as follows:

$$
u=W x \rightarrow x=W^{-1} u
$$

where $W$ denotes the un-mixing matrix as the spatial filter for independence component (IC) estimation, $W^{-1}$ denotes the $n \times$ $n$ component mixing matrix whose columns contain the relative weights of back-projected components to each of the scalp channels.

The portion of the original EEG data forms the $i$-th independent component $\left(x_{\mathrm{i}}\right)$, a product of the two vectors, as follows:

$$
x_{i}=W_{i}^{-1} u_{i}
$$

The whole data $(x)$ are the sum of the back-projected ICs $\left(x_{\mathrm{i}}\right)$, as follows:

$$
x=\sum_{i}^{n} x_{i}
$$

By mapping these weights to the corresponding EEG channels on the head model, we can visualize the scalp map projection (scalp map) of each source components.

\section{Features Extraction}

For feature extraction, a power spectral density (PSD) was applied with the autoregressive (AR) modelling for generating the frequency spectrum. This paper used the Burg method of the AR spectral estimation which has been used in the EEG application as reported in [15]. The PSD of the AR Burg estimation is calculated in the following equation: 


$$
\hat{P}_{B U R G}(\omega)=\frac{\hat{e}_{p}}{\left|1+\sum_{k=1}^{P} \hat{a}_{p}(k) e^{-j k \omega}\right|^{2}}
$$

where $\hat{P}_{B U R G}(\omega)$ denotes the PSD of the AR Burg estimation, $\hat{a}_{p}(k)$ denotes the estimated $\mathrm{AR}$ parameters and $\hat{e}_{p}$ denotes the reflection coefficients.

The order of AR was set as 25 in this study. The PSD was applied to $2 \mathrm{~s}$ of each EEG segment, equivalent to 512 points for $256 \mathrm{~Hz}$ of sampling rate. Applying PSD, the time domain of EEG data segment was converted into frequency domain. A total of 4 EEG bands including delta band $(0.5-3 \mathrm{~Hz})$, theta band $(3.5-7.5 \mathrm{~Hz})$, alpha band $(8-13 \mathrm{~Hz})$ and beta band $(13.5-$ $30 \mathrm{~Hz}$ ), were used. The total PSD for each EEG band was calculated based on the numerical integration of trapezoidal rule, which provided 4 units of power values. As a result, 24 units of power for 6 EEG channels were used and 8 units of power for 2 EEG channels were used.

\section{E. Classification}

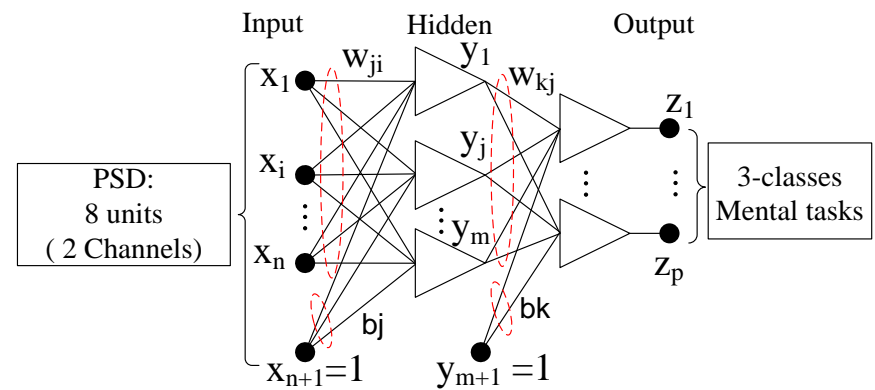

Fig. 3. The neural network structure in this study

Multilayer perceptron neural network (MLP) depicted in Fig.3 is used as a classifier. The Levenberg-Marquardt with Bayesian regularization algorithm is applied to train the classifier. According to Bayesian framework [16, 17], the performance function that contains regularization terms is expressed in following equation:

$$
F(x)=\beta E_{E}+\alpha E_{W}
$$

where $\alpha$ and $\beta$ are hyperparameters, and the ratio $\alpha / \beta$ controls the effective complexity of the network solution.

By introducing hyperparameters in the performance function, neural network weights can be prevented to be too large that result in poor generalization for new test cases. As a result, a validation set is not required in a neural network training procedure. The Bayesian regularization algorithm is applied to update hyperparameters as follows:

$$
\alpha^{M P}=\frac{\gamma}{2 E_{W}\left(x^{M P}\right)} ; \beta^{M P}=\frac{N-\gamma}{2 E_{D}\left(x^{M P}\right)}
$$

where $\gamma=n-2 \alpha^{M P} \operatorname{tr}\left(H^{M P}\right)^{-1}$ is called the effective number of parameters, $n$ is the total number of parameters in the network, $N$ is the total number of errors, and $H$ is Hessian matrix of $F(\underline{x})$ at the minimum point of $x^{M P}$. The log evidence of model $H_{i}$, is evaluated by Bayesian framework as follows:

$$
\begin{aligned}
& \ln p\left(D \mid H_{i}\right)=-\alpha_{M P} E_{W}^{M P}-\beta_{M P} E_{W}^{M P}-\frac{1}{2} \ln |A| \\
& +\frac{W}{2} \ln \alpha_{M P}+\frac{N}{2} \ln \beta_{M P}+\ln M !+2 \ln M \\
& +\frac{1}{2} \ln \frac{2}{\gamma}+\frac{1}{2} \ln \frac{2}{N-\gamma}
\end{aligned}
$$

where $W$ is the number of network parameters, and $M$ is the number of hidden nodes. The optimal network structure is the network that has the highest evidence.

\section{RESULTS}

Using EEGLAB, FastICA was applied to the 6 EEG channels data from 10 participants for each mental task to obtain six source components. To estimate the location of the equivalent dipoles for generating IC scalp map, an inverse source modelling was applied. The projected scalp map with the selected dominant feature component is shown in Fig. 4 and Table I, respectively. The six EEG channels covered the left central channel (C3), right central channel (C4), left parietal channel (P3), right parietal channel (P4), left occipital channel (O1) and right occipital channel (O2).
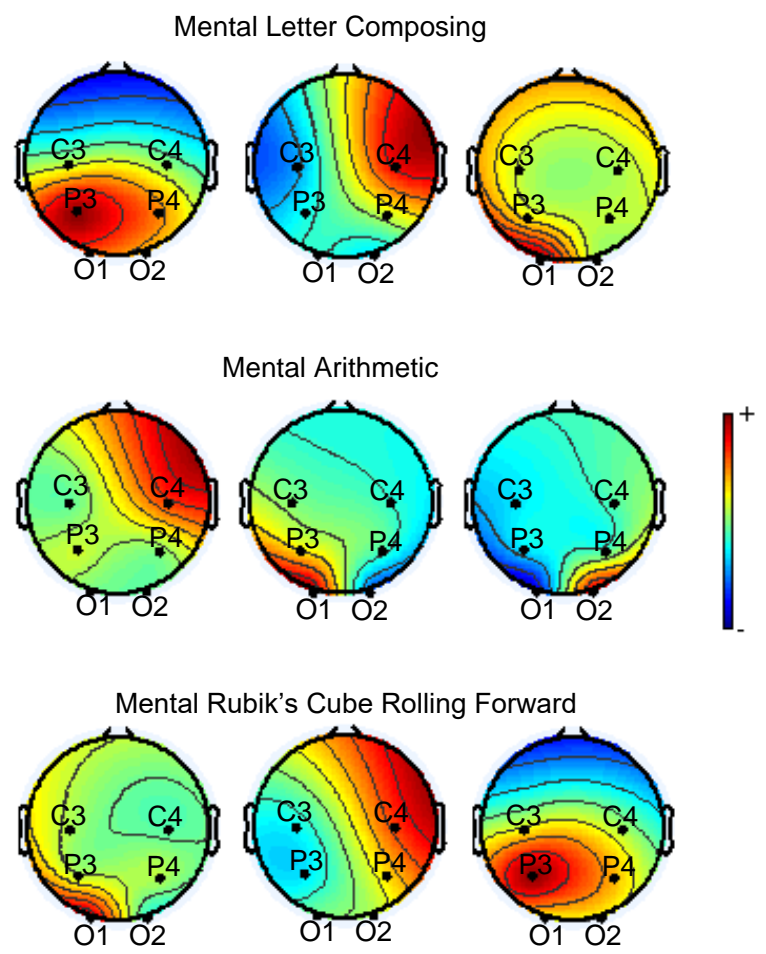

Fig. 4. The scalp map of the dominant features for three mental tasks 
TABLE I. SELECTED EEG CHANNELS OF THE DOMINANT FEATURES

\begin{tabular}{|c|c|}
\hline \hline Mental Tasks & $\begin{array}{c}\text { Selected Channels of } \\
\text { Dominant Features }\end{array}$ \\
\hline $\begin{array}{c}\text { Mental Letter } \\
\text { Composing }\end{array}$ & $\mathrm{C} 4, \mathrm{P} 3, \mathrm{O} 1$ \\
\hline Mental Arithmetic & $\mathrm{C} 4, \mathrm{O} 1, \mathrm{O} 2$ \\
\hline $\begin{array}{c}\text { Rubik's cube } \\
\text { rolling forward }\end{array}$ & $\mathrm{C} 4, \mathrm{P} 3, \mathrm{O} 1$ \\
\hline \hline
\end{tabular}

Specifically, for mental letter composing, the dominant features were found in $\mathrm{C} 4, \mathrm{P} 3$ an $\mathrm{O} 1$ channels; for mental arithmetic calculation, the dominant features were found in $\mathrm{P} 3$, O1 and O2 channels; for imagining mental Rubik's cube rolling forward, the dominant features were found $\mathrm{C} 4, \mathrm{P} 3$ and O1 channels. As a result, the dominant features for the three mental tasks were found on the scalp map in the location of the left hemisphere, including the left parietal channel (P3) and the left occipital channel (O1). For the right hemisphere, the dominant features of three mental tasks were found on the right central channel (C4) and right occipital channel (O2). Therefore, by analyzing the dominant features showing in the scalp map, the EEG channels can be selected and reduced from original 6 channels $(\mathrm{C} 3, \mathrm{C} 4, \mathrm{P} 3, \mathrm{P} 4, \mathrm{O} 1, \mathrm{O} 2)$ into 4 channels (P3, O1, C4 and O2).

Moreover, as shown in Table I, for the left hemisphere channels, the O1 channel contributes in three mental tasks. P3 channel contributes in two mental tasks (letter composing and Rubik's cube rolling).For the right hemisphere channels, C4 channel dominates in three mental tasks. The $\mathrm{O} 2$ channel only dominates in the mental arithmetic. If selecting the best pair of channel of the left and right channel group, O1 and $\mathrm{C} 4$ is the chosen pair.

A two-channel classification was applied to the chosen four channels (P3, O1, C4 and O2) selected from the ICA scalp map. This could be divided into the left group (P3 and O1 channels) and the right group ( $\mathrm{C} 4$ and $\mathrm{O} 2$ channels). As a result, four-combinations of the two channels, found from two groups (P3\&C4, P3\&O2, O1\&C4 and O1\&O2), were obtained. The size of the matrix feature was $24 \times 4100$ units for from each mental task. With the total of three mental tasks, this gave feature data set with the dimension of $8 \times 12300$ units. For the Bayesian neural network classification, the data set was divided into training set with $50 \%$ of the overall sets and the remaining portion was used for the testing set.

The classification result of the two EEG channel combinations is shown in Fig.5. The best two channels classification is O1\&C4 with an accuracy of $76.4 \%$, the second best channels classification is $\mathrm{P} 3 \& \mathrm{O} 2$ with an accuracy of $74.5 \%$, the third best channel classification is $\mathrm{P} 3 \& \mathrm{C} 4$ with an accuracy of $71.9 \%$ and the last pair, $\mathrm{O} 1 \& \mathrm{O} 2$ provides classification an accuracy of $70 \%$. The plot of the log evidence against the optimum number of the Bayesian neural network hidden nodes was 26 for two channels O1\&C4 is shown in Fig.6.

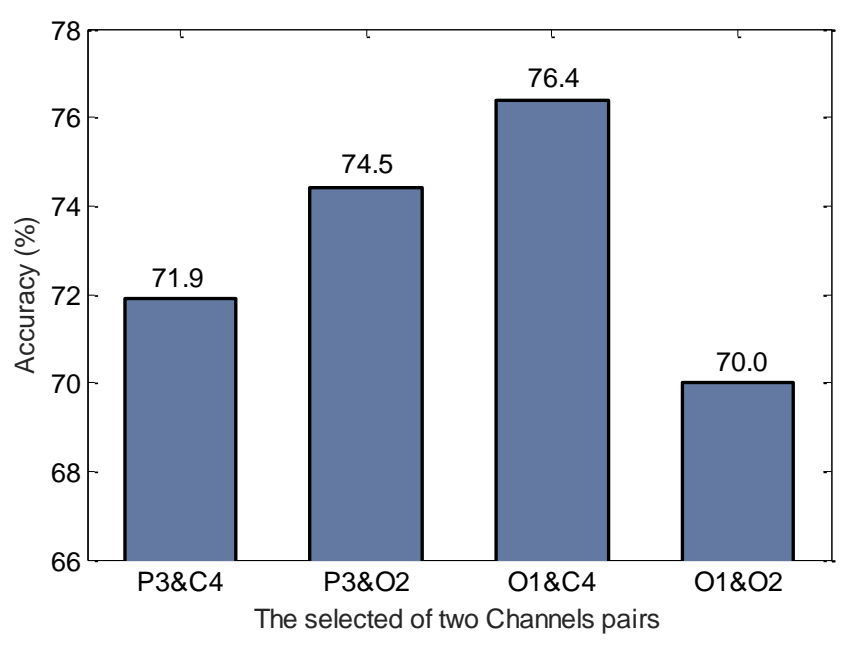

Fig. 5. Two EEG channel classification for selected channels

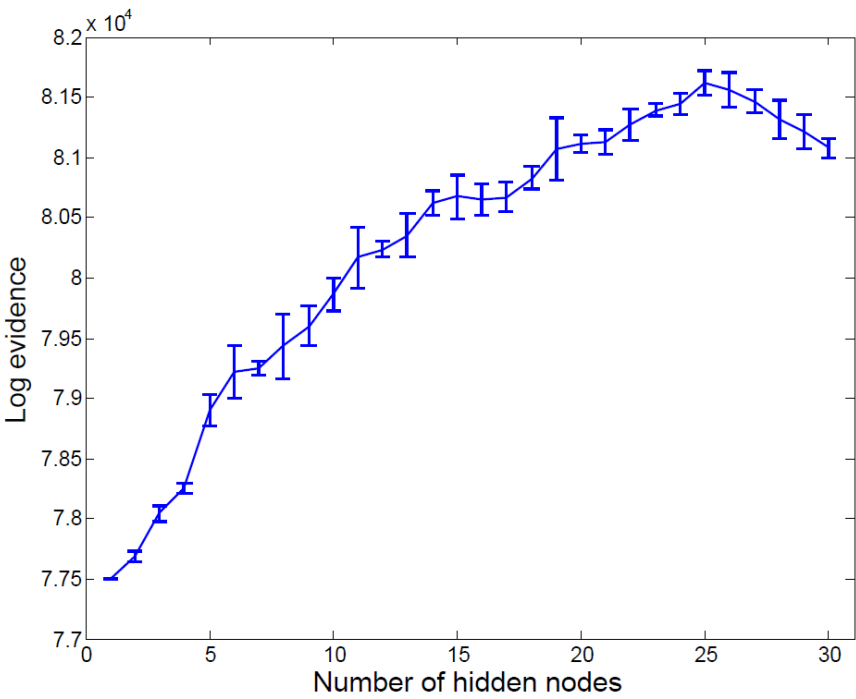

Fig. 6. Log evidence for the Bayesian neural network classifier for two cTwo EEG channel (C4\&O1)

\section{CONCLUSION}

In this paper, the ICA method has been applied to select the optimal EEG channels for a three mental task classification (letter composing, arithmetic, Rubik's cube rolling) with the experiment data recorded from 10 participants. After ICA composition with its back-projected scalp map being applied, there were 4 EEG channels dominantly contributing to the three mental tasks, including $\mathrm{P} 3 \& \mathrm{O} 1$ (from right group channels) and $\mathrm{C} 4 \& \mathrm{O} 2$ (from left group channels). The combination of $\mathrm{O} 1 \& \mathrm{C} 4$ was found as the best two channel pair. The result classification on combinations selected two EEG channels have also been conducted with the feature extractor based on the PSD and Bayesian neural network as classifier. The obtained results showed that the best two EEG channels is $\mathrm{O} 1 \& \mathrm{C} 4$ with an accuracy of $76.4 \%$, followed by $\mathrm{P} 3 \& \mathrm{O} 2$ with an accuracy of $74.5 \%$; $\mathrm{P} 3 \& \mathrm{C} 4$ with an accuracy of $71.9 \%$ and $\mathrm{O} 1 \& \mathrm{O} 2$ with an accuracy of $70 \%$. The accuracy 
of the best pair classification also verified the best pair channel chosen from the scalp map projection. This proved the capability of the ICA of selecting the optimal EEG channels for the mental task-based BCI classification.

\section{REFERENCES}

[1] J. Wolpaw and E. W. Wolpaw, Brain-computer interfaces: principles and practice: Oxford University Press, 2012.

[2] B. He, S. Gao, H. Yuan, and J. R. Wolpaw, "Brain-computer interfaces," in Neural Engineering: Springer, 2013, pp. 87-151.

[3] D. A. Craig, H. T. Nguyen, and H. A. Burchey, "Two Channel EEG Thought Pattern Classifier," in The 28th Annual International Conference of the IEEE Engineering in Medicine and Biology Society (EMBC) 2006, pp. 1291-1294.

[4] D. J. McFarland, W. A. Sarnacki, and J. R. Wolpaw, "Electroencephalographic (EEG) control of three-dimensional movement," Journal of Neural Engineering, vol. 7, p. 036007, 2010.

[5] P. Boord, A. Craig, Y. Tran, and H. Nguyen, "Discrimination of left and right leg motor imagery for brain-computer interfaces," Medical \& Biological Engineering \& Computing, vol. 48, pp. 343-350, 2010/04/01 2010.

[6] M. Conson, S. Sacco, M. Sarà, F. Pistoia, D. Grossi, and L. Trojano, "Selective motor imagery defect in patients with locked-in syndrome," Neuropsychologia, vol. 46, pp. 2622-2628, 2008.

[7] D. A. Craig and H. T. Nguyen, "Adaptive EEG Thought Pattern Classifier for Advanced Wheelchair Control," in The 29th Annual International Conference of the IEEE Engineering in Medicine and Biology Society (EMBC), 2007, pp. 2544-2547.

[8] R. Chai, S. H. Ling, G. P. Hunter, Y. Tran, and H. T. Nguyen, "Brain-Computer Interface Classifier for Wheelchair Commands Using Neural Network With Fuzzy Particle Swarm Optimization," IEEE Journal of Biomedical and Health Informatics, vol. 18, pp. 1614-1624, 2014.

[9] A. Delorme and S. Makeig, "EEGLAB: an open source toolbox for analysis of single-trial EEG dynamics including independent component analysis," Journal of Neuroscience Methods, vol. 134, pp. 9-21, 2004.

[10] S. Makeig, A. J. Bell, T.-P. Jung, and T. J. Sejnowski, "Independent component analysis of electroencephalographic data," Advances in neural information processing systems, pp. 145-151, 1996.

[11] H. T. Nguyen, "Intelligent technologies for real-time biomedical engineering applications," International Journal of Automation and Control, vol. 2, Nos.2/3, pp. 274-285, 2008.

[12] R. Chai, S. H. Ling, G. P. Hunter, Y. Tran, and H. T. Nguyen, "Classification of wheelchair commands using brain computer interface: comparison between able-bodied persons and patients with tetraplegia," in The 35th Annual International Conference of the IEEE Engineering in Medicine and Biology Society (EMBC), 2013, pp. 989-992.

[13] G. H. Klem, H. O. Lüders, H. Jasper, and C. Elger, "The ten-twenty electrode system of the International Federation," Electroencephalogr Clin Neurophysiol, vol. 52, p. 3, 1999.
[14] A. Hyvärinen and E. Oja, "Independent component analysis: algorithms and applications," Neural networks, vol. 13, pp. 411-430, 2000.

[15] D. J. McFarland and J. R. Wolpaw, "Sensorimotor rhythm-based brain-computer interface (BCI): model order selection for autoregressive spectral analysis," Journal of Neural Engineering, vol. 5, p. 155,2008 .

[16] C. M. Bishop, "Neural networks for pattern recognition," 1995.

[17] D. J. MacKay, "A practical Bayesian framework for backpropagation networks," Neural computation, vol. 4, pp. 448-472, 1992. 